\title{
La mediación escolar en los planes y programas institucionales de convivencia en España
}

\author{
School mediation in institutional plans and \\ programmes of school coexistence in Spain \\ María-Isabel VIANA-ORTA \\ Universidad de Valencia
}

Recibido: Febrero 2013

Aceptado: Mayo 2013

\begin{abstract}
Resumen
Las primeras experiencias de mediación escolar en España datan de mediados de la década de los noventa. País Vasco, Cataluña y Madrid fueron las comunidades autónomas pioneras en la implementación de programas de mediación y, desde entonces, sigue extendiéndose por todo el territorio nacional, siendo cada día más numerosos los centros educativos que disponen de un servicio de mediación. A su vez, la mayoría de comunidades autónomas - en uso de sus competencias educativas- elaboraron, durante la primera década de 2000 , sus propias normas y sus propios planes o programas institucionales de convivencia escolar. Simultáneamente, en algunas comunidades también se firmaron acuerdos o pactos sociales a favor de una cultura de paz y de una convivencia pacífica en los centros educativos de sus respectivos territorios. En esta línea, el objetivo central de este artículo es realizar un estudio comparado para conocer qué comunidades autónomas han apostado por la mediación escolar al incluirla en los acuerdos y pactos sociales suscritos y en sus propios planes y programas institucionales de convivencia.
\end{abstract}

Palabras clave: Mediación; convivencia escolar; programas de convivencia; resolución de conflictos; educación comparada.

\begin{abstract}
The first school mediation experiences in Spain date back to the mid-nineties. The Basque country, Catalonia and Madrid were the first autonomous regions in introducing school mediation programmes and since then, school mediation is extending all over the country. At the same time, the great majority of autonomous regions developed their school coexistence regulations, plans and programmes during the first decade from 2000. Simultaneously, some of those regions signed social agreements to promote a culture of peace at schools in its own territories. In the same vain, the main aim of this article is to carry out a comparative study to find out which autonomous regions support school mediation, including it in the social agreements that they have signed, as well as in its plans, and coexistence institutional programmes.
\end{abstract}

Keywords: Mediation; school coexistence; coexistence programmes; conflict resolution; comparative education. 
La mediación utilizada en el ámbito educativo tiene sus orígenes en Estados Unidos en la década de los años sesenta y se va extendiendo por todo el mundo (Van Slyck \& Stern, 1996; Suares, 1996; Alzate, 1999; Halligan \& Araiz, 1999; Johnson \& Johnson, 1999). En España, las primeras experiencias se producen en la década de los noventa de la mano de profesores pioneros con conocimientos de otras lenguas o con experiencias educativas en otros países $\mathrm{y}$, también, por grupos ya iniciados en la investigación de otro tipo de conflictos, como es el caso del Centro de Resolución de Conflictos Gernika Gogoratuz. País Vasco, Cataluña y Madrid, son las comunidades que primero implementaron programas de mediación escolar en nuestro país en los años 1993, 1996 y 1997, respectivamente (Uranga, 1997.; Torrego, 2003; Boqué, 2003; Torrego \& Galán, 2008; Casanovas, Magre \& Lauroba, 2010; Viana-Orta, 2011). A raíz de estas iniciativas, se produce una extensión rápida y desigual de la mediación escolar por numerosos centros educativos del territorio nacional.

Posteriormente, en la primera década de 2000 , se produce la regulación normativa de la convivencia escolar en el estado español. Por un lado, a nivel nacional, se aprueban algunas leyes, se firma un pacto social y se elaboran diversos planes de convivencia. A su vez, por otro lado, las diferentes comunidades autónomas (CCAA) en uso de sus competencias en materia de educación- fueron aprobando su propia normativa, suscribiendo sus propios pactos sociales y elaborando sus propios planes y programas de convivencia.

Así, a nivel nacional y en el marco de la Década Internacional para la Cultura de Paz (2001-2010) proclamada por las Naciones Unidas (Resolución 53/25, noviembre 1998), se aprueba la Ley 27/2005, de 30 de noviembre, de fomento de la educación y la cultura de paz (Mayor \& De la Corte, 2002; De la Corte, Blanco \& Sabucedo, 2002). Esta ley establece una serie de medidas destinadas al ámbito educativo y de la investigación, con el objeto de establecer la cultura de paz y no-violencia en nuestra sociedad. Entre estas medidas, se dispone que corresponde al Gobierno "promover la formación especializada de hombres y mujeres en técnicas de resolución de conflictos, negociación y mediación (art. 2.7). Con posterioridad, se aprobó la Ley Orgánica 2/2006, de 3 de mayo, de Educación (LOE), que considera tanto un principio (art. 1.k) como un fin (art. 2.1.c) del sistema educativo español, "la prevención de conflictos y la resolución pacífica de los mismos". Podemos apreciar, pues, que la LOE no apuesta de forma clara y decidida por la mediación escolar porque no la contempla de forma expresa en su texto aunque, obviamente, podemos entenderla incluida de forma implícita junto a cualesquiera otras medidas que puedan adoptarse para la "resolución pacífica de conflictos".

A su vez, a nivel nacional, se elabora una serie de "Propuestas para un Pacto Social y Político por la Educación", que establecen los objetivos de la educación para la década 2010-2020, entre los que se contempla expresamente la mediación escolar (Objetivo 10, Propuesta 105) apoyando su creación en todos los centros educativos y promoviendo también el acuerdo en este sentido de las administraciones educativas autonómicas. Además, en 2006, el Ministerio de Educación suscribe dos acuerdos de mejora de la convivencia escolar. El primero de ellos, fue el "Plan para la Promoción y Mejora de la Convivencia Escolar", suscrito en marzo por el Ministerio y las 
organizaciones sindicales, cuyo objetivo era la mejora de la convivencia y la prevención de la violencia escolar y que se concibió desde el diálogo y el consenso con las CCAA y la implicación de sus principales destinatarios: profesorado, alumnado y familias. El segundo, fue el "Plan Director para la Convivencia y la Seguridad Escolar", suscrito entre el Ministerio de Educación y el Ministerio de Interior en diciembre. Este Acuerdo Marco manifestaba un firme compromiso de los poderes públicos y del resto de agentes sociales para la erradicación del entorno escolar de cualquier conducta violenta y reconocía la eficacia probada de la mediación escolar como mecanismo de prevención de la violencia en el ámbito educativo.

\section{Objetivos de la investigación}

En este contexto nacional, los objetivos perseguidos por la presente investigación fueron:

- Realizar una recopilación de los pactos sociales suscritos por las diferentes CCAA en sus respectivos territorios, así como de los planes y programas institucionales de convivencia escolar puestos en marcha por las mismas.

- Realizar un estudio comparado para conocer en cuáles de todos esos documentos se incluyó expresamente la mediación escolar y, en su caso, en qué términos, para poder apreciar el diferente grado de respaldo institucional que está recibiendo por parte de las CCAA.

Es necesario destacar, pues, que quedó fuera de nuestros objetivos en el presente trabajo, un estudio comparado del respaldo normativo recibido por las diferentes CCAA, para centrarnos solo en lo que hemos denominado respaldo institucional, es decir, el realizado a través de pactos sociales y de planes y programas institucionales (Viana-Orta, 2011).

\section{Materiales y metodología}

En nuestro estudio comparado partimos del método comparativo deductivo que fue presentado por Bereday y Hilker, y que luego recibió aportaciones de García Garrido, Ferrer y Martínez, entre otros (García-Garrido, 1990; Ferrer, 2002; Martínez, 2003). Siguiendo este método, las etapas fundamentales en las que vamos a sintetizar aquí nuestro estudio son: fase descriptiva, fase de yuxtaposición y fase comparativa.

Las unidades comparativas del estudio fueron la totalidad de las comunidades autónomas españolas (CCAA) $(n=17)$, y la variable que se comparó fue los documentos institucionales (pactos sociales, planes y programas) relacionados con la convivencia escolar firmados, aprobados y puestos en marcha por cada una de ellas. Así pues, todos esos documentos fueron las fuentes de información que utilizamos. 


\section{Resultados y discusión}

\section{Fase descriptiva}

\section{Andalucía}

Andalucía se sumó a la "Declaración y Plan de Acción de la Asamblea de las Naciones Unidas del Decenio Internacional de una Cultura de Paz y Noviolencia para los niños del mundo", con la firma del "Manifiesto Andaluz a favor de la Cultura de Paz y Noviolencia". Este documento, declara la voluntad de todos los firmantes (educadores, artistas, deportistas, escritores, políticos, ayuntamientos, asociaciones, sindicatos, etc.) de, entre otras, participar activamente en la creación de condiciones de convivencia, buscando soluciones creativas a los conflictos, aunque no hace ninguna referencia expresa a la mediación.

Por su parte, el "Plan Andaluz de Educación para la Cultura de Paz y Noviolencia", hecho público por la Orden de 25 de julio de 2002, sí que contempla expresamente la mediación al proponer, entre otras, las siguientes medidas:

- Formación del profesorado para la puesta en marcha de estrategias de regulación y mediación de conflictos en el centro.

- Puesta en marcha de programas de arbitraje y mediación de conflictos en el centro.

- Creación de la figura del profesor/a mediador/a en aquellos centros que pos sus características especiales lo precisen.

Además, Andalucía cuenta también desde el curso 2002-2003, con una convocatoria anual para los centros que quieran incorporarse a la Red "Escuela: Espacio de Paz", aunque este programa tampoco contempla la mediación escolar de forma expresa.

\section{Aragón}

El Gobierno de Aragón lleva a cabo, desde el curso 2005-2006, diferentes actuaciones dentro de su Plan de Convivencia, entre las que queremos destacar la "Guía Cuento contigo", en la que se contempla la mediación escolar llevada a cabo por el tutor como medida para favorecer la convivencia entre iguales en el aula.

Además, la administración educativa y los representantes de la comunidad educativa firmaron en febrero de 2008 el "Acuerdo para la Mejora de la Convivencia Escolar en los centros educativos de Aragón", entre cuyos objetivos destacamos el de "promover e incentivar el uso de procesos de participación, mediación, formación y diálogo como recursos para la prevención, detección y resolución pacífica de los conflictos en los centros escolares". Entre las líneas de actuación propuestas para su consecución, destacamos las siguientes:

- Elaboración de protocolos para la resolución de conflictos que deberán especificar, entre otros elementos, la responsabilidad de funciones de mediación y la formación en mediación de todos los sectores (medida $\mathrm{n}^{\mathrm{o}} 3$ ).

- Por lo que respecta al asesoramiento a los centros escolares en materia de conflicto escolar se declara la intención de constituir en cada Servicio 
Provincial una Asesoría que podrá intervenir en procesos de mediación y de formación de los sectores de la comunidad educativa (medida $\mathrm{n}^{\mathrm{o}} 7$ ).

- Con el fin de favorecer la adquisición y desarrollo de habilidades sociales y técnicas de mediación en la resolución de conflictos se declara la intención de la Administración educativa de impulsar de forma periódica la realización de actividades de formación dirigidas a representantes y miembros de los sectores de la comunidad educativa (medida $\mathrm{n}^{\circ} 12$ ).

\section{Asturias}

En diciembre de 2006 se firma el "Acuerdo Social para la Mejora de la Convivencia Escolar" en el Principado de Asturias, entre cuyos objetivos destacamos el de "favorecer la mediación como metodología más adecuada para la solución pacífica de conflictos en los centros educativos". Entre las acciones e iniciativas establecidas por el Acuerdo destacamos, además:

- Incluir en la formación permanente del profesorado los contenidos orientados la prevención de los conflictos, a la mejora de la convivencia y a la práctica de la mediación escolar.

- Poner en práctica estrategias de implicación del alumnado en la prevención y la solución de los conflictos de convivencia, fomentando los programas de mediación como medida favorecedora de la resolución pacífica de conflictos.

Además, en Asturias se celebran los encuentros escolares por la convivencia "Encuentrastur" en los que colabora la Consejería de Educación del Principado, cuyo objetivo es la formación de todos los miembros de la comunidad educativa en estrategias pacíficas de resolución de conflictos en contextos escolares. Queremos destacar que, aunque la mediación está incluida en la formación, no aparece recogida de forma expresa en el documento.

\section{Baleares}

La Administración educativa de las Islas Baleares en colaboración con el Instituto para la Convivencia y el Éxito Escolar y con el objetivo de crear una cultura de mediación que permita una mejora integral del clima escolar, incorporó los denominados "Programas de Competencia Social y Mediación Escolar" para la Educación Secundaria siguiendo el ejemplo de Cataluña. En base a ellos, en una primera fase, a lo largo del curso 2007-2008 se realizó la formación de un grupo de formadores en competencia social y en mediación escolar con la intención de que en el curso siguiente ayudaran, formaran y guiaran a los centros interesados en crear servicios de mediación a través de actividades de formación en centros. Así, a partir del curso 2008-2009 los centros que imparten Educación Secundaria Obligatoria y que estén interesados, disponen de una convocatoria anual de proyectos de innovación pedagógica de "Creación y puesta en funcionamiento de un servicio de mediación escolar".

\section{Canarias}

En el caso de la Comunidad Autónoma de Canarias, ni el "Plan Canario de Educación para la Convivencia" ni el documento "Medidas implantadas por la 
Consejería de Educación, Universidades, Cultura y Deportes, para la prevención y mejora de la convivencia escolar", recogen ninguna referencia expresa a la mediación escolar. Sin embargo, la "Propuesta de Programa de Mediación de conflictos en el ámbito educativo", responde a una decidida voluntad de institucionalización, potenciación y extensión de la mediación en los centros escolares de Canarias, y en la que se declara que la Administración Educativa fomentará el uso de la mediación en todos los contextos educativos en que resulte viable y, en especial, en la etapa de Educación Secundaria Obligatoria.

\section{Cantabria}

El Plan de Convivencia para los Centros Educativos de Cantabria recoge, potencia y estructura las buenas prácticas que se realizan en los centros educativos y en él se proponen actuaciones dirigidas a todos los miembros de la comunidad educativa y a todos los sectores sociales, sin embargo, carece de un documento marco propiamente dicho por lo que no hemos podido constatar la referencia expresa o no a la mediación escolar.

\section{Castilla-La Mancha}

El "Acuerdo por la Convivencia en los centros escolares de Castilla-La Mancha" fue firmado en 2006 a iniciativa del Presidente de Castilla-La Mancha y de las Cortes Regionales mediante resolución de estas de 27 de abril del mismo año. Dicho acuerdo fue suscrito por los representantes del alumnado, del profesorado, de las familias, de los sindicatos, de los medios de comunicación y de otras instituciones sociales y culturales. Su finalidad es la de apoyar y desarrollar iniciativas que ayuden a fomentar y a reforzar las buenas relaciones de convivencia en los centros docentes, con el compromiso de colaboración de toda la sociedad castellano-manchega. Entre las iniciativas y acciones que propone, destacamos por su relación directa con la mediación escolar, las siguientes:

- La elección por el Consejo Escolar del centro de un miembro adulto de la comunidad educativa que ejerza las funciones de mediación y arbitraje en los procesos más importantes de conflictividad que surjan entre los miembros de su comunidad educativa, ofreciendo medidas alternativas a la vía judicial o a los procesos meramente sancionadores. Todos los miembros de la comunidad educativa deben asumir su compromiso de reconocimiento y respeto a las decisiones de mediación y arbitraje que adopte quien sea elegido para tal fin, y sin que ello suponga menoscabo de las acciones judiciales o administrativas reconocidas en las leyes y en la normativa básica en materia de educación ( $\mathrm{n}^{\circ}$ 4).

- El impulso en todos los centros de las actuaciones de los equipos de mediación, y el fomento de la mediación escolar como principio educativo $\left(\mathrm{n}^{\mathrm{0}} 5\right)$.

- La mejora de la formación inicial del profesorado, incorporando los contenidos orientados a la prevención de la conflictividad y a la práctica de la convivencia y la mediación escolar $\left(\mathrm{n}^{\mathrm{o}} 6\right)$.

- El desarrollo de planes de formación para las familias relacionadas con la convivencia, la solución pacífica de los conflictos y la mediación $\left(n^{\circ} 12\right)$. 
- La organización de talleres y cursos para formar a los alumnos en los procesos de mediación y solución pacífica de conflictos, para que así puedan asumir de una forma más eficaz el protagonismo que les corresponde en estos procesos $\left(\mathrm{n}^{\circ}\right.$ 13).

\section{Castilla y León}

En la Comunidad Autónoma de Castilla y León, no hemos encontrado ningún documento que describa un Plan Autonómico de Convivencia Escolar aunque sí que se hace referencia a él en diferentes lugares y normas. Por ello, interpretamos que debemos entender por tal, todas las iniciativas que se están llevando a cabo desde la Consejería de Educación con el objetivo de promocionar la convivencia escolar y de prevenir la violencia. En este sentido, destacamos tres programas de apoyo a la convivencia, aunque ninguno de los tres hace mención expresa a la mediación escolar:

- Programa "IESocio" que es una herramienta que utilizando las nuevas tecnologías permite recoger información para evaluar las relaciones entre compañeros midiendo distintos indicadores sobre convivencia y métodos sociométricos.

- Programa "Fondo documental convivencia escolar" iniciado en 2005 con el objetivo de apoyar la actuación del profesorado mediante la dotación de recursos.

- Programa "Protección y asistencia jurídica al profesorado y alumnado" que funciona desde diciembre de 2006.

\section{Cataluña}

La Comunidad Autónoma de Cataluña, apuesta de forma clara y decidida por la mediación escolar. El "Proyecto de Convivencia y Éxito Educativo" cuenta, entre sus objetivos generales, con el de fomentar la cultura de mediación como herramienta básica de gestión de conflictos. Por su parte, el "Programa de Convivencia y Mediación Escolar" está dirigido tanto a Educación Infantil y Primaria como a Educación Secundaria. En el caso de la Secundaria el programa se subdivide, a su vez, en dos: Programa de Competencia Social y Programa de Mediación Escolar.

\section{Comunidad Valenciana}

En la Comunidad Valenciana, el "Plan de Prevención de la Violencia y Promoción de la Convivencia en los centros escolares de la Comunidad Valenciana" (Plan PREVI), fue concebido para dar respuesta a las necesidades referentes a problemas de convivencia detectados. El Plan incluye medidas de prevención y proporciona herramientas y recursos a los docentes que les ayuden a trabajar dentro de sus aulas y en sus centros, pero no hace ninguna referencia expresa a la mediación escolar.

\section{Extremadura}

En Extremadura, la Consejería de Educación y las organizaciones sindicales firmaron en 2006 el "Compromiso Social por la Convivencia", en el que se declaraba la intención de potenciar la mediación escolar. Por su parte, el "Plan regional de la Convivencia escolar en Extremadura 2007”, incluyó entre las actuaciones previstas 
para mejorar la convivencia en los centros el establecimiento de la mediación para la resolución pacífica de los conflictos. Además afirma, que el objetivo de la mediación va más allá de la adopción de acuerdos y que se trata de una oportunidad de fortalecer a los individuos y sus relaciones.

Además, Extremadura cuenta con la "Red Extremeña de Escuelas por una cultura de Paz, Igualdad y Noviolencia" y con el "Pacto Social por la educación" firmado en junio de 2009, aunque ninguno de los dos documentos incluye expresamente la mediación escolar.

\section{Galicia}

En esta Comunidad Autónoma, el "Plan Integral de Mejora de la Convivencia Escolar en Galicia", sí que hace referencia expresa a la mediación escolar. Por un lado, establece la posibilidad de que el Reglamento de Régimen Interno de cada centro incorpore en el mismo programa de mediación y, por otro, la incluye dentro de la formación permanente del profesorado.

\section{La Rioja}

En el caso de la Rioja, entendemos como un plan de convivencia autonómico, todas las actuaciones llevadas a cabo por la administración educativa para el fomento de la convivencia escolar que son anunciadas desde su portal web pero a cuya información no se puede acceder y, entre las cuales, no hay ninguna directamente relacionada con la mediación escolar.

\section{Madrid}

El Pleno de la Asamblea de Madrid, el 20 de abril de 2006, aprobó la Resolución no 14/2006 por la que se instaba al Gobierno de la Comunidad de Madrid a elaborar un plan por la convivencia en los centros educativos en el que debían aparecer iniciativas y actuaciones para hacer frente a cuestiones como el acoso escolar o a cualquier tipo de discriminación. El plan debía conllevar, entre otros aspectos, los siguientes:

- La creación de un Foro por la Convivencia en el seno del Consejo Escolar de la Comunidad de Madrid.

- La revisión de la normativa de convivencia en los centros docentes y del Reglamento de Organización y Funcionamiento de los Centros.

- Impulsar la elaboración en los centros educativos de Planes específicos de convivencia.

- Reforzar la autoridad del profesor.

- Establecimiento de convenios con el Gobierno de la Nación para coordinar acciones y recursos.

Además, se instaba al Gobierno a escuchar las aportaciones de todos los sectores implicados: organizaciones sindicales, grupos políticos, organizaciones de padres y madres, organizaciones de estudiantes, otros colectivos sociales (inmigrantes, mujeres...), Consejo de la Juventud de la Comunidad de Madrid y el Defensor del Menor. Queremos destacar que, en ninguna de las iniciativas puestas en marcha (Foro, revisión normativa...) hemos encontrado referencia alguna a la mediación. 
Por su parte, el Programa "Convivir es vivir" para el desarrollo de la convivencia y prevención de la violencia escolar es concebido como un programa educativo, interinstitucional, abierto y preventivo cuyos objetivos son mejorar los niveles de convivencia en los centros educativos y en su entorno, fomentar actitudes de respeto y tolerancia para prevenir la aparición de actos violentos dentro y fuera de la institución escolar y proporcionar al profesorado habilidades y estrategias de intervención ante posibles conflictos que puedan surgir en los centros relacionados con el deterioro de la convivencia. El Programa se inició en 1997 ofreciendo una convocatoria anual pero en la convocatoria para participar en el programa no hay tampoco ninguna referencia expresa a la mediación escolar.

\section{Murcia}

En Murcia, el "Pacto Social por la Educación en la Región de Murcia, 2009-2012", es el tercero adoptado por la sociedad murciana desde que se realizaron los traspasos competenciales en materia educativa a esta comunidad. Los dos anteriores tuvieron lugar en 1998 y en 2005. El objetivo de este Pacto es el establecimiento de planes de mejora de la educación en su camino hacia el éxito y la excelencia educativa. Entre las líneas de calidad contempladas en el mismo, se hace referencia a la mediación escolar en dos ocasiones:

- Se formula como objetivo potenciar las funciones del Observatorio de la Convivencia, facilitando la recogida de los datos para su posterior análisis y estudio de los mismos en relación con la mejora de la convivencia y la mediación en la Región de Murcia.

- Se deben desarrollar actuaciones de formación del profesorado que le capacite para, entre otras, ejecutar planes de mejora de la convivencia y mediación y de reducción del absentismo escolar.

Pero ni el "Plan Regional sobre Convivencia Escolar en la Comunidad Autónoma de la Región de Murcia", aprobado por el pleno del Observatorio en julio de 2009, ni en el "Programa Educando para la justicia", desarrollado conjuntamente por el Consejo General de Poder Judicial, el Tribunal Superior de Justicia y la Consejería de Educación, encontramos referencia alguna a la mediación escolar.

\section{Navarra}

En la Comunidad Foral de Navarra también debemos entender como plan o programa autonómico de convivencia todas las actuaciones e iniciativas adoptadas y encaminadas hacia la mejora de la convivencia escolar porque no hemos encontrado un documento marco. Entre las actuaciones emprendidas, la Resolución 632/2005, de 5 de julio, estableció el Plan de Mejora de la Convivencia en los centros educativos de la Comunidad Foral de Navarra y a su amparo se constituyó la Asesoría de Convivencia encuadrada en el Departamento de Educación de Navarra y con funciones muy similares a los Observatorios para la Convivencia Escolar. Por otra parte, la Consejería de Educación realiza campañas anuales a favor de la convivencia escolar aunque queremos destacar que no hay ninguna directamente relacionada con la mediación escolar. 


\section{País Vasco}

En la Comunidad Autónoma del País Vasco se puso en marcha el primer "Programa de Educación para la Convivencia" en el año 2000, y a partir de entonces se ha ido ampliando y profundizando hasta la actualidad. Por su parte, el Departamento de Educación, Universidades e Investigación, a través de los Programas de Innovación Educativa, fija las líneas prioritarias para la mejora de los procesos educativos. Las Líneas Prioritarias de Innovación Educativa 2007-2010 establecen un marco general que gira en torno a tres ejes: una escuela para todos y todas, una escuela mejor y una escuela en la sociedad del siglo XXI.

Entre los programas del primer eje hemos encontrado referencias al aprendizaje de la resolución pacífica de conflictos pero ninguna referencia expresa a la mediación. Del eje 2, el programa "Educar para la Convivencia, la Paz y los Derechos Humanos", sí que incluye, entre las propuestas que formula a los centros escolares para impulsar y desarrollar la educación para la convivencia y la paz, la de plantear el respeto mutuo, el diálogo, la negociación y la mediación como principales procedimientos para encauzar y resolver los conflictos y ofrecer, cuando sea preciso, un servicio de mediación en los conflictos. Este programa da paso al Plan Vasco de Educación para la Paz y los Derechos Humanos (2008-2011) que prevé la creación del Consejo Consultivo de Educación en Derechos Humanos y por la Paz que pasa, en 2011, a llamarse Consejo Consultivo de Convivencia Democrática y Deslegitimación de la Violencia. En cualquier caso, no se le atribuye ninguna función directamente relacionada con la mediación.

Cabe matizar en este sentido que, para el curso 2010-2011, el "Plan Vasco de Educación para la Paz y los Derechos Humanos" se reformula en el plan "Convivencia democrática y deslegitimación de la violencia", cuyas actuaciones estratégicas se dirigen a comprometer el currículum pero también la organización de los centros y la formación del profesorado en aras a que se vivan y se practiquen en los centros los derechos humanos y la paz con especial hincapié en la convivencia democrática y la deslegitimación de la violencia. Sin embargo, no se hace ninguna referencia expresa a la mediación.

Dentro de este marco, encontramos también el programa de "Educación para la Convivencia y la Paz" es un programa destinado a toda la comunidad educativa con carácter abierto y flexible que permita a los centros elaborar colectivamente un proyecto para favorecer la convivencia, pero tampoco hace referencia expresa a la mediación escolar.

Por lo tanto, la Comunidad Autónoma del País Vasco tiene actualmente varios programas institucionales relacionados con la convivencia en los centros educativos pero ninguno de ellos está directamente relacionado con la mediación escolar, ni la incluye expresamente. 


\section{Fase de yuxtaposición y conclusiones de la yuxtaposición}

\section{Fase de yuxtaposición}

En la Tabla 1 podemos observar de forma yuxtapuesta la totalidad de las CCAA $(\mathrm{n}=17)$; la existencia o no, en cada caso, de pactos o acuerdos sociales y de planes o programas institucionales; $y$, finalmente, si hay o no en cada uno de ellos alguna referencia expresa a la mediación escolar y, en su caso, el nivel de implicación con la misma.

\begin{tabular}{|c|c|c|}
\hline & \multicolumn{2}{|c|}{$\begin{array}{l}\text { LA MEDIACIÓN ESCOLAR EN LOS PLANES O PROGRAMAS } \\
\text { INSTITUCIONALES DE CONVIVENCIA ESCOLAR EN ESPAÑA }\end{array}$} \\
\hline $\begin{array}{l}\text { UNIDADES } \\
\text { COMPARATIVAS }\end{array}$ & $\begin{array}{c}\text { PACTOS SOCIALES, PLANES } \\
\text { INSTITUCIONALES O } \\
\text { PROGRAMAS } \\
\text { INSTITUCIONALES } \\
\end{array}$ & $\begin{array}{l}\text { RELACIÓN DEL DOCUMENTO CON } \\
\text { LA MEDIACIÓN ESCOLAR }\end{array}$ \\
\hline \multirow{3}{*}{ ANDALUCÍA } & $\begin{array}{l}\text { Manifiesto Andaluz a favor de la } \\
\text { Cultura de Paz y Noviolencia. }\end{array}$ & No hace referencia expresa a la mediación. \\
\hline & $\begin{array}{l}\text { Plan Andaluz de Educación para la } \\
\text { Cultura de Paz y Noviolencia. }\end{array}$ & $\begin{array}{l}\text { Propone la formación del profesorado en } \\
\text { mediación, la puesta en marcha de } \\
\text { programas de mediación y la creación de la } \\
\text { figura del profesor/a mediador/a en aquellos } \\
\text { centros que por sus características } \\
\text { especiales lo precisen. }\end{array}$ \\
\hline & Red "Escuela: Espacio de Paz". & No hace referencia expresa a la mediación. \\
\hline \multirow[t]{2}{*}{ ARAGÓN } & $\begin{array}{l}\text { Acuerdo para la Mejora de la } \\
\text { Convivencia Escolar en los centros } \\
\text { educativos de Aragón. }\end{array}$ & $\begin{array}{l}\text { - Entre los objetivos: promover e incentivar } \\
\text { el uso de la mediación. } \\
\text { - Entre las líneas de actuación: elaboración } \\
\text { de protocolos de responsabilidades y } \\
\text { funciones en temas de mediación, impulsar } \\
\text { de forma periódica actividades de } \\
\text { formación en mediación de todos los } \\
\text { sectores, constitución de una Asesoría en } \\
\text { cada Servicio Provincial para intervenir en } \\
\text { procesos de mediación y de formación. }\end{array}$ \\
\hline & $\begin{array}{l}\text { Plan de Convivencia Cuento } \\
\text { Contigo. }\end{array}$ & $\begin{array}{l}\text { Contempla la mediación escolar pero } \\
\text { llevada a cabo por el tutor como una } \\
\text { medida para favorecer la convivencia entre } \\
\text { iguales en el aula. }\end{array}$ \\
\hline \multirow[t]{2}{*}{ ASTURIAS } & $\begin{array}{l}\text { Acuerdo Social para la Mejora de la } \\
\text { Convivencia Escolar. }\end{array}$ & $\begin{array}{l}\text { - Entre los objetivos: favorecer la } \\
\text { mediación como la metodología más } \\
\text { adecuada para la solución pacífica de } \\
\text { conflictos en los centros educativos. } \\
\text { - Entre las acciones: incluir la práctica de la } \\
\text { mediación escolar en la formación } \\
\text { permanente del profesorado y fomentar los } \\
\text { programas de mediación. }\end{array}$ \\
\hline & $\begin{array}{l}\text { Encuentros escolares por la } \\
\text { Convivencia "Encuentrastur". }\end{array}$ & $\begin{array}{l}\text { Su objetivo es la formación en estrategias } \\
\text { pacíficas de conflictos aunque no hace } \\
\text { referencia expresa a la mediación. }\end{array}$ \\
\hline BALEARES & $\begin{array}{l}\text { Programas de Competencia Social y } \\
\text { Mediación Escolar. }\end{array}$ & $\begin{array}{l}\text { Tienen como objetivo crear una cultura de } \\
\text { mediación que permita una mejora integral }\end{array}$ \\
\hline
\end{tabular}




\begin{tabular}{|c|c|c|}
\hline & & $\begin{array}{l}\text { del clima escolar. En este sentido los } \\
\text { centros disponen de una convocatoria anual } \\
\text { de proyectos de innovación pedagógica de } \\
\text { "Creación y puesta en funcionamiento de } \\
\text { un servicio de mediación escolar". }\end{array}$ \\
\hline \multirow[b]{2}{*}{ CANARIAS } & $\begin{array}{l}\text { Plan Canario de Educación para la } \\
\text { Convivencia. }\end{array}$ & No hace referencia expresa a la mediación. \\
\hline & $\begin{array}{l}\text { Propuesta de Programa de } \\
\text { Mediación de conflictos en el } \\
\text { ámbito educativo. }\end{array}$ & $\begin{array}{l}\text { Supone una decidida voluntad de } \\
\text { institucionalización, potenciación y } \\
\text { extensión de la mediación educativa en los } \\
\text { centros escolares y declara que la } \\
\text { Administración Educativa en Canarias } \\
\text { fomentará el uso de la mediación en todos } \\
\text { los contextos educativos en que resulte } \\
\text { viable y, en especial, en la etapa de } \\
\text { Educación Secundaria Obligatoria. }\end{array}$ \\
\hline CANTABRIA & $\begin{array}{l}\text { Plan Regional para la Convivencia } \\
\text { en los centros escolares de } \\
\text { Cantabria. }\end{array}$ & $\begin{array}{l}\text { Recoge, potencia y estructura las buenas } \\
\text { prácticas que se realizan en los centros } \\
\text { educativos y se proponen actuaciones } \\
\text { dirigidas a todos los miembros de la } \\
\text { comunidad educativa y a todos los sectores } \\
\text { sociales, sin embargo, no hemos encontrado } \\
\text { ninguna referencia expresa a la mediación } \\
\text { escolar entre dichas propuestas. }\end{array}$ \\
\hline $\begin{array}{l}\text { CASTILLA LA } \\
\text { MANCHA }\end{array}$ & $\begin{array}{l}\text { Acuerdo por la convivencia en los } \\
\text { centros escolares de Castilla-La } \\
\text { Mancha. }\end{array}$ & $\begin{array}{l}\text { - Elección por el Consejo Escolar de centro } \\
\text { de un miembro adulto de la comunidad } \\
\text { educativa que ejerza las funciones de } \\
\text { mediación y arbitraje en los procesos más } \\
\text { importantes de conflictividad que surjan } \\
\text { entre los miembros de su comunidad } \\
\text { educativa, ofreciendo medidas alternativas } \\
\text { a la vía judicial o a los procesos meramente } \\
\text { sancionadores. } \\
\text { - Impulso en todos los centros de las } \\
\text { actuaciones de los equipos de mediación, y } \\
\text { el fomento de la mediación escolar como } \\
\text { principio educativo. } \\
\text {-Mejora de la formación inicial del } \\
\text { profesorado, incorporando los contenidos } \\
\text { orientados a la prevención de la } \\
\text { conflictividad y a la práctica de la } \\
\text { convivencia y la mediación escolar. } \\
\text { - Desarrollo de planes de formación para las } \\
\text { familias relacionadas con la convivencia, la } \\
\text { solución pacífica de los conflictos y la } \\
\text { mediación. } \\
\text { - Organización de talleres y cursos para } \\
\text { formar a los alumnos en los procesos de } \\
\text { mediación y solución pacífica de conflictos. }\end{array}$ \\
\hline $\begin{array}{l}\text { CASTILLA Y } \\
\text { LEÓN }\end{array}$ & $\begin{array}{l}\text { No hemos encontrado ningún } \\
\text { documento o normativa que describa } \\
\text { un Plan Autonómico de Convivencia }\end{array}$ & $\begin{array}{l}\text { Ninguno de los programas de apoyo a la } \\
\text { convivencia que hemos encontrado } \\
\text { (Programa "IESocio", Programa "Fondo }\end{array}$ \\
\hline
\end{tabular}




\begin{tabular}{|c|c|c|}
\hline & $\begin{array}{l}\text { Escolar, parece que debemos } \\
\text { entender por tal, todas las iniciativas } \\
\text { que se están llevando a cabo desde } \\
\text { la Consejería de Educación con el } \\
\text { objetivo de promocionar la } \\
\text { convivencia escolar y de prevenir la } \\
\text { violencia. }\end{array}$ & $\begin{array}{l}\text { documental convivencia escolar" y } \\
\text { Programa "Protección y asistencia jurídica } \\
\text { al profesorado y al alumnado") hace } \\
\text { referencia expresa a la mediación escolar. }\end{array}$ \\
\hline \multirow{2}{*}{ CATALUÑA } & $\begin{array}{l}\text { Proyecto de Convivencia y Éxito } \\
\text { Educativo. }\end{array}$ & $\begin{array}{l}\text { Objetivo general: fomentar la cultura de la } \\
\text { mediación como herramienta básica de } \\
\text { gestión de conflictos. }\end{array}$ \\
\hline & $\begin{array}{l}\text { Programa de Convivencia y } \\
\text { Mediación Escolar. }\end{array}$ & $\begin{array}{l}\text { Objetivo: formar en la convivencia } \\
\text { fomentando las relaciones positivas y la } \\
\text { resolución pacífica de conflictos en el seno } \\
\text { de cada centro. }\end{array}$ \\
\hline $\begin{array}{l}\text { COMUNIDAD } \\
\text { VALENCIANA }\end{array}$ & $\begin{array}{l}\text { Plan de Prevención de la Violencia y } \\
\text { Promoción de la Convivencia en los } \\
\text { centros escolares de la Comunidad } \\
\text { Valenciana (Plan PREVI). }\end{array}$ & No hace referencia expresa a la mediación. \\
\hline \multirow{3}{*}{ EXTREMADURA } & $\begin{array}{l}\text { Compromiso Social por la } \\
\text { Convivencia. }\end{array}$ & $\begin{array}{l}\text { Intención de potenciar la mediación escolar } \\
\text { como procedimiento conciliado. }\end{array}$ \\
\hline & $\begin{array}{l}\text { Plan Regional de la Convivencia } \\
\text { Escolar en Extremadura } 2007 .\end{array}$ & $\begin{array}{l}\text { Incluye entre las actuaciones previstas para } \\
\text { mejorar la convivencia en los centros el } \\
\text { establecimiento de la mediación para la } \\
\text { resolución pacífica de conflictos y la } \\
\text { entiende como una oportunidad de reforzar } \\
\text { a los individuos y sus relaciones (su } \\
\text { objetivo no es alcanzar acuerdos sino } \\
\text { orientar las relaciones). }\end{array}$ \\
\hline & $\begin{array}{l}\text { Red Extremeña de Escuelas por una } \\
\text { Cultura de Paz, Igualdad y } \\
\text { Noviolencia. }\end{array}$ & $\begin{array}{l}\text { La orden de convocatoria hace referencia } \\
\text { continua al arreglo pacífico de los } \\
\text { conflictos pero ninguna referencia expresa } \\
\text { a la mediación. }\end{array}$ \\
\hline GALICIA & $\begin{array}{l}\text { Plan Integral de Mejora de la } \\
\text { Convivencia Escolar en Galicia, } \\
\text { 2007-2010. }\end{array}$ & $\begin{array}{l}\text { - Los centros pueden incorporar al RRI } \\
\text { programas de mediación. } \\
\text { - Incluir entre la formación permanente del } \\
\text { profesorado, la resolución de conflictos y la } \\
\text { mediación. }\end{array}$ \\
\hline LA RIOJA & $\begin{array}{l}\text { No hemos encontrado ningún } \\
\text { documento o normativa que describa } \\
\text { un Plan Autonómico de Convivencia } \\
\text { Escolar, parece que debemos } \\
\text { entender por tal, todas las iniciativas } \\
\text { que se están llevando a cabo desde } \\
\text { la Consejería de Educación con el } \\
\text { objetivo de promocionar la } \\
\text { convivencia escolar y de prevenir la } \\
\text { violencia. }\end{array}$ & $\begin{array}{l}\text { Entre las medidas adoptadas no hay } \\
\text { ninguna relacionada con la mediación } \\
\text { escolar. }\end{array}$ \\
\hline
\end{tabular}




\begin{tabular}{|c|c|c|}
\hline \multirow[t]{2}{*}{ MADRID } & $\begin{array}{l}\text { Resolución } \mathrm{N}^{\circ} 14 / 2006 \text { del Pleno de } \\
\text { la Asamblea de Madrid, instando al } \\
\text { Gobierno elaborar planes contra el } \\
\text { acoso escolar y a promover y } \\
\text { desarrollar actuaciones relativas al } \\
\text { fomento de la convivencia y al } \\
\text { respeto en todos los centros docentes } \\
\text { de la Comunidad de Madrid (BOCM } \\
\left.\mathrm{n}^{\mathrm{o}} 153,27-04-2006\right) \text {. }\end{array}$ & No hace referencia expresa a la mediación. \\
\hline & Programa "Convivir es vivir". & No hace referencia expresa a la mediación. \\
\hline \multirow[t]{3}{*}{ MURCIA } & $\begin{array}{l}\text { Pacto Social por la Educación en la } \\
\text { Región de Murcia 2009-2012. }\end{array}$ & $\begin{array}{l}\text { - Objetivo: potenciar las funciones del } \\
\text { Observatorio de la Convivencia, facilitando } \\
\text { la recogida de datos en relación con la } \\
\text { mejora de la convivencia y la mediación. } \\
\text { - Formación del profesorado en planes de } \\
\text { mejora de la convivencia, mediación y } \\
\text { reducción del absentismo escolar. }\end{array}$ \\
\hline & $\begin{array}{l}\text { Plan Regional sobre Convivencia } \\
\text { Escolar en la Comunidad Autónoma } \\
\text { de la Región de Murcia. }\end{array}$ & No hace referencia expresa a la mediación. \\
\hline & Programa "Educando en la justicia". & $\begin{array}{l}\text { No es un programa relacionado } \\
\text { directamente con la mediación pero desde } \\
\text { su enlace web se ofrecen también ejemplos } \\
\text { de mediaciones en el ámbito educativo. }\end{array}$ \\
\hline NAVARRA & $\begin{array}{l}\text { No hemos encontrado en la } \\
\text { Comunidad Foral de Navarra un } \\
\text { Plan o Programa Autonómico de } \\
\text { Convivencia propiamente dicho sino } \\
\text { que debemos entender como tal } \\
\text { todas las acciones emprendidas para } \\
\text { la mejora de la convivencia, entre } \\
\text { las que se encuentran campañas } \\
\text { anuales a favor de la convivencia } \\
\text { escolar. }\end{array}$ & $\begin{array}{l}\text { Ninguna de las campañas realizadas está } \\
\text { directamente relacionada con la mediación. }\end{array}$ \\
\hline \multirow[t]{2}{*}{ PAÍS VASCO } & $\begin{array}{l}\text { El Programa "Educar para la } \\
\text { Convivencia, la Paz y los Derechos } \\
\text { Humanos" dio paso al Plan Vasco de } \\
\text { Educación para la Paz y los } \\
\text { Derechos Humanos (2008-2011) y } \\
\text { éste, a su vez, se reformula en el } \\
\text { plan "Convivencia democrática y } \\
\text { deslegitimación de la violencia". }\end{array}$ & $\begin{array}{l}\text { El Programa original "Educar para la } \\
\text { Convivencia, la Paz y los Derechos } \\
\text { Humanos" incluía, entre las propuestas } \\
\text { realizadas a los centros, la de plantear el } \\
\text { respeto mutuo, el diálogo, la negociación y } \\
\text { la mediación como principales } \\
\text { procedimientos para encauzar y resolver los } \\
\text { conflictos y la de ofrecer, cuando sea } \\
\text { preciso, un servicio de mediación en los } \\
\text { conflictos, pero no los actuales programas. }\end{array}$ \\
\hline & $\begin{array}{l}\text { Programa de Educación para la } \\
\text { Convivencia y la Paz en los centros } \\
\text { escolares de la CAPV, } 2006 .\end{array}$ & No hace referencia expresa a la mediación. \\
\hline
\end{tabular}

Tabla 1. La mediación en los planes y programas institucionales de convivencia escolar en España. 


\section{Conclusiones de la yuxtaposición}

Queremos recordar que, dentro de la variable comparada "documentos institucionales (pactos, planes y programas) relacionados con la convivencia escolar y firmados, aprobados y puestos en marcha por las diferentes CCAA" y, tal y como podemos observar en la tabla, hemos incluido documentos que podrían clasificarse en tres apartados distintos: 1) acuerdos, manifiestos o pactos sociales relacionados con una cultura de paz o con la convivencia escolar; 2) planes institucionales de convivencia escolar; y 3) programas específicos relacionados con la convivencia escolar. Nos interesaba conocer, como ya hemos apuntado, si cada una de las CCAA contempla la mediación escolar de forma expresa, al menos, en alguno de los tres planos mencionados, lo que supondría un primer nivel de respaldo institucional a la misma.

Del análisis se desprende que el 58,82\% de las CCAA ( $\mathrm{n}=10$ ) sí que contemplan la mediación escolar de forma expresa, al menos, en alguno de los tres tipos de documentos analizados. Estas CCAA son: Andalucía; Aragón; Asturias; Baleares; Canarias; Castilla-La Mancha; Cataluña; Extremadura; Galicia; y Murcia. Queremos llamar la atención, tal y como podemos observar en la tabla, que existen grandes diferencias en la forma de aludir a la mediación escolar de los diferentes documentos, y así, nos encontramos CCAA con una clara y decidida defensa del uso de procesos mediadores en los centros educativos y, otras, cuya inclusión no es tan decidida.

Dentro del $41,18 \%$ restante ( $n=7)$, es decir, entre aquellas CCAA que no incluyen ninguna referencia expresa a la mediación escolar en sus documentos institucionales, se encuentran: Cantabria; Castilla y León; Comunidad Valenciana; La Rioja; Madrid; Navarra; y País Vasco.

\section{Conclusiones}

En primer lugar, podemos concluir que en España se empezaron a implementar programas de mediación escolar en la década de los noventa y que las CCAA pioneras en su implementación fueron País Vasco (1993), Cataluña (1996) y Madrid (1997). A partir de entonces, la mediación escolar se extiende por numerosos centros educativos del territorio nacional.

En segundo lugar, durante la primera década de 2000 en España, se producen numerosas iniciativas relacionadas con la convivencia escolar, tanto a nivel nacional promovidas por el Ministerio de Educación y con aplicación en todo el territorio, como a nivel autonómico, promovidas por todas las CCAA, en virtud de sus propias competencias educativas.

En tercer lugar, y en relación a la anterior, a nivel nacional y en el marco de la Década Internacional para la Cultura de Paz (2001-2010) promovido por las Naciones Unidas, se aprueba la Ley $27 / 2005$, de 30 de noviembre, de fomento de la educación y cultura de paz, que incluye expresamente la necesidad de formar a hombres y mujeres en técnicas de resolución de conflictos, negociación y mediación. Se pone en marcha, también, el "Plan Director para la Convivencia y la Seguridad Escolar" que reconoce la 
eficacia probada de la mediación escolar como mecanismo de prevención de la violencia en el ámbito educativo. Por último, el documento "Propuestas para un Pacto Social y Político por la Educación", incluye entre los objetivos de la educación para la década 2010-2020, la mediación escolar apoyándola y promoviendo también el acuerdo en este sentido de las administraciones educativas autonómicas.

En cuarto lugar, a nivel autonómico, entre las iniciativas promovidas relacionadas con la convivencia escolar, y como resultado de los documentos analizados, sin haber entrado en el presente trabajo a analizar la normativa reguladora de la convivencia escolar, podemos concluir que en España, el 58,82 \% de las CCAA ( $\mathrm{n}=10)$ sí que contempla la mediación escolar de forma expresa en sus documentos institucionales relacionados con la convivencia escolar como son Pactos Sociales, Planes Institucionales o Programas Institucionales, frente a un $41,18 \%$ restante $(n=7)$ que no lo hace. Las CCAA que sí lo hacen son: Andalucía, Aragón, Asturias, Baleares, Canarias, Castilla-La Mancha, Cataluña, Extremadura, Galicia y Murcia. Las que no, son: Cantabria, Castilla y León, Comunidad Valenciana, La Rioja, Madrid, Navarra y el País Vasco.

En quinto lugar, de entre las CCAA que sí que contemplan expresamente la mediación escolar, queremos destacar algunas cuestiones:

- Hay un 29,41\% de CCAA ( $n=5$ ) que la recogen en los Pactos Sociales suscritos por la mismas y, por lo tanto, defendidos por diversos sectores de la sociedad y no solo por la administración (Aragón, Asturias, Castilla-La Mancha, Extremadura y Murcia). Podemos observar que el hecho de contemplarse en los Pactos Sociales, no siempre ha condicionado a la administración a recogerla en sus planes o programas.

- Hay un $17,64 \%$ de CCAA ( $n=3)$ que la contemplan desde sus Planes de Convivencia (Andalucía, Aragón y Galicia) y, como podemos observar, salvo Aragón, no son coincidentes con las que lo hacían en los Pactos Sociales.

- Hay un 17,64\% de CCAA $(n=3)$ que tienen programas específicos de mediación escolar (Baleares, Canarias y Cataluña) y que tampoco coinciden con las anteriores, es decir, con las CCAA que incluyen la mediación en Pactos Sociales o en Planes Institucionales de Convivencia.

- Queremos destacar también, el diferente grado de compromiso adquirido con la mediación escolar en todos los documentos analizados que es más intenso y decidido cuando se trata de programas específicos y no de meras declaraciones de intenciones como las recogidas en pactos sociales y en planes institucionales.

En sexto y último lugar, de entre las CCAA que no contemplan la mediación escolar de forma expresa, queremos destacar también algunas cuestiones:

- Sorprende que dos de las CCAA pioneras en la aplicación de programas de mediación en España, como son País Vasco y Madrid, no se refieran a la mediación escolar de forma expresa en ninguno de sus documentos institucionales.

- También queremos llamar la atención en el hecho de que, a pesar de que estas CCAA no hacen mención expresa a la mediación escolar en sus documentos 
institucionales, nos consta el uso de procesos mediadores y la existencia de equipos de mediación en centros educativos de todas ellas.

Será interesante, pues, continuar nuestras investigaciones en esta línea de conocer la realidad de los centros educativos en relación con el uso de la mediación escolar en los diferentes territorios.

\section{Referencias bibliográficas}

ALZATE, R. (1999). Enfoque global de la escuela como marco de aplicación de los programas de resolución de conflictos. En Brandoni, F. (Comp.). Mediación escolar. Propuestas, reflexiones y experiencias. (pp. 31-55). Buenos Aires: Paidós.

BINABURO, J.A. y MUÑOZ, B. (2007). Educar desde el conflicto. Guía para la mediación escolar. Barcelona: Ceac.

BOQUÉ, M.C. (2003). Cultura de mediación y cambio social. Barcelona: Gedisa.

CASANOVAS, P., MAGRE, J. \& LAUROBA, M.E. (Dir.) (2010). Llibre Blanc de la Mediació a Catalunya. Barcelona: Generalitat de Catalunya.

CONSEJERÍA DE EDUCACIÓN DE ASTURIAS. Acuerdo Social para la Mejora de la Convivencia Escolar. Consultado el 14-05-2013 en: http://www.educastur.es/index.php?option=com_content\&task=view\&id=889\&Ite $\mathrm{mid}=182$

CONSEJERÍA DE EDUCACIÓN DE ASTURIAS. Encuentros escolares por la Convivencia "Encuentrastur". Consultado el 10-05-2013 en:

http://blog.educastur.es/convivencia/2011/06/07/encuentrastur-2011/

CONSEJERÍA DE EDUCACIÓN DE CANARIAS. Plan Canario de Educación para la Convivencia. Consultado el 14-05-2013 en:

http://www.gobiernodecanarias.org/educacion/WebDGOIE/scripts/default.asp?Id Sitio $=13 \&$ Cont $=155 \&$ categoria $=3294$

CONSEJERÍA DE EDUCACIÓN DE CANARIAS. Propuesta de Programa de Mediación de conflictos en el ámbito educativo. Consultado el 16-01-2011 en: http://www.gobiernodecanarias.org/educacion/udg/convivencia/content/articulo5/ promocion/PROPUESTA\%20PROGRAMA\%20MEDIACIÓN.doc

CONSEJERÍA DE EDUCACIÓN DE CANTABRIA. Plan para la convivencia en los centros escolares de Cantabria. Consultado el 14-05-2013 en: http://www.educantabria.es/informacion_institucional/publicaciones/plan-para-laconvivencia-en-los-centros-escolares-de-cantabrian2005 
CONSEJERÍA DE EDUCACIÓN DE CASTILLA-LA MANCHA. Acuerdo por la Convivencia en los centros escolares de Castilla-La Mancha. Consultado el 1005-2013 en:

http://www.educa.jccm.es/educa-

$\mathrm{jccm} / \mathrm{cm} /$ educa_jccm $/ \mathrm{tkContent}$ ?idContent $=30053 \&$ locale $=$ es_ES\&textOnly $=$ fals e

CONSEJERÍA DE EDUCACIÓN DE LA COMUNIDAD DE MADRID. Programa Convivir es vivir. Consultado el 12-05-2013 en: http://www.madrid.org/dat_capital/upe/supe_convivir.htm

CONSEJERÍA DE EDUCACIÓN DE LA REGIÓN DE MURCIA. Pacto Social por la Educación en la Región de Murcia, 2009-2012. Consultado el 14-05-2013 en:

http:/www.carm.es/web/pagina?IDCONTENIDO=4954\&IDTIPO=246\&RASTR $\mathrm{O}=\mathrm{c} 943 \$ \mathrm{~m} 4331,4330$

CONSEJERÍA DE EDUCACIÓN DE LA REGIÓN DE MURCIA. Plan Regional sobre Convivencia Escolar en la Comunidad Autónoma de la Región de Murcia. Consultado el 14-05-2013 en: http:/www.carm.es/web/pagina?IDCONTENIDO=5316\&IDTIPO=100\&RASTR $\mathrm{O}=\mathrm{c} 792 \$ \mathrm{~m} 4001$

CONSEJERÍA DE EDUCACIÓN DE LA REGIÓN DE MURCIA. Programa Educando en la justicia. Consultado el 14-05-2013 en:

http:/www.educarm.es/portal/admin/webForm.php?aplicacion=PROGRAMAS_E 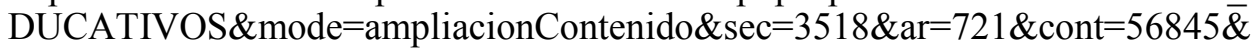 $\mathrm{web}=102 \&$ zona $=$ PROFESORES\&menuSeleccionado $=183$

CONSELLERÍA D'EDUCACIÓ DE LA COMUNITAT VALENCIANA. Plan de Prevención de la Violencia y de Promoción de la Convivencia en los centros escolares de la Comunidad Valenciana (Plan PREVI). Consultado el 12-05-2013 en: http://www.edu.gva.es/eva/val/previ.htm

CONSEJERÍA DE EDUCACIÓN DE EXTREMADURA. Compromiso Social por la Convivencia y Plan Regional de la Convivencia Escolar en Extremadura. Consultado el 14-05-2013 en: http://recursos.educarex.es/pdf/convivencia/plan_regional_convivencia.pdf

CONSEJERÍA DE EDUCACIÓN DE EXTREMADURA. Red Extremeña de Escuelas por una Cultura de Paz, Igualdad y Noviolencia. Consultado el 12-05-2013 en: http:/www.educarex.es/web/guest/redes-escuelas-de-cultura-y-paz

CONSEJERÍA DE EDUCACIÓN DE LA RIOJA. Portal de Convivencia Escolar y Atención a casos de Acoso Escolar. Consultado el 10-05-2013 en: http:/www.educarioja.org/educarioja/html/alm/acoso_escolar/acoso_escolar.html

CONSELLERÍA D’EDUCACIÓ DE LES ILLES BALEARS. Programas de Competencia Social y Mediación Escolar. Consultado el 12-05-2013 en: 
http://www.caib.es/sacmicrofront/archivopub.do?ctrl=MCRST151ZI30887\&id=3 0887

DE LA CORTE, L., BLANCO, A. \& SABUCEDO, J.M. (2002). El artificio de la violencia. Fundamentos y obstáculos para el diseño de una cultura de paz y de derechos humanos. Cultura y Educación, 14 (4), 373-390.

DEPARTAMENTO DE EDUCACIÓN DE ARAGÓN. Acuerdo para la Mejora de la Convivencia Escolar en los centros educativos de Aragón. Consultado el 12-052013 en:

http://ryc.educa.aragon.es/Atencion_Diversidad/docs/Acuerdo_Convivencia_18\% 2002\%2008.pdf

DEPARTAMENTO DE EDUCACIÓN DE NAVARRA. Portal web de la Asesoría para la convivencia. Consultado el 06-02-2011 en: http://www.educacion.navarra.es/portal/Informacion+de+Interes/Asesoria+para+1 a + Convivencia

DEPARTAMENTO DE EDUCACIÓN DEL PAÍS VASCO. Programa de Educación para la Convivencia y la Paz en los centros escolares de la Comunidad Autónoma del País Vasco. Consultado el 12-05-2013 en: http://www.hezkuntza.ejgv.euskadi.net/r43-

573/es/contenidos/informacion/dig2/es_5614/adjuntos/bakea/ca_indice.htm

DEPARTAMENTO DE EDUCACIÓN DEL PAÍS VASCO. Convivencia democrática $y$ deslegitimación de la violencia. Consultado el 12-05-2013 en: http://www.eskolabakegune.euskadi.net/web/guest/planes-de-convivencia

DEPARTAMENT D'ENSENYAMENT DE CATALUNYA. Proyecto de Convivencia y Éxito Educativo. Consultado el 14-05-2013 en: http://www20.gencat.cat/docs/Educacio/Home/Arees_actuacio/innovacio_educati $\mathrm{va} / \mathrm{Pla} \% 20 \mathrm{de} \% 20$ convivencia/doc_marc_projecte_convivencia.pdf

DEPARTAMENT D'ENSENYAMENT DE CATALUNYA. Programa de Convivencia y Mediación Escolar. Consultado el 17-01-2011 en: http://www.xtec.cat/innovacio/convivencia/

DEPARTAMENTO DE EDUCACIÓN DE ARAGÓN. Plan de Convivencia Cuento Contigo. Consultado el 12-05-2013 en:

http://www.educaragon.org/files/Cuento\%20contigo_mod1\%20.pdf

FARRÉ, S. (2009). Gestión de conflictos: taller de mediación. Un enfoque socioafectivo. Barcelona: Ariel.

FERRER, F. (2002). La educación comparada actual. Barcelona: Ariel.

GARCÍA-GARRIDO, J.L. (1990). Fundamentos de la Educación Comparada. (3 ${ }^{\mathrm{a}}$ ed.). Madrid: Dykinson.

HALLIGAN, J. \& ARAIZ, R. (1999). Los beneficios y desafíos de la aplicación de currículos para la Resolución de Conflictos en las escuelas. En Brandoni, F. 
(Comp.). Mediación escolar. Propuestas, reflexiones y experiencias. (pp. 75-94). Buenos Aires: Paidós.

JOHNSON, D.W. \& JOHNSON, R.T. (1999). Los alumnos como pacificadores: cómo enseñar a los estudiantes a resolver conflictos. En Brandoni, F. (Comp.). Mediación escolar. Propuestas, reflexiones y experiencias. (pp. 95-121). Buenos Aires: Paidós.

JUNTA DE ANDALUCÍA. Manifiesto Andaluz a favor de la Cultura de Paz y Noviolencia. Consultado el 10-05-2013 en:

http:/www.juntadeandalucia.es/averroes/impe/web/contenido?pag=/contenidos/B/ InnovacionEInvestigacion/ProyectosInnovadores/EscuelaEspacioDePaz/Seccion/ ManifiestoAndaluz\&\&idSeccion $=29505$

JUNTA DE ANDALUCÍA. Plan Andaluz de Educación para la Cultura de Paz y Noviolencia. Consultado el 17-02-2011 en:

http://www.juntadeandalucia.es/educacion/convivencia

JUNTA DE ANDALUCÍA. Red "Escuela: Espacio de Paz". Consultado el 12-052013

en:

http://www.juntadeandalucia.es/educacion/convivencia/com/jsp/listado.jsp?canal= $711 \&$ seccion $=$ centros

JUNTA DE CASTILLA Y LEÓN. Portal de convivencia de Castilla y León. Consultado el 14-05-2013 en: http://www.educa.jcyl.es/convivencia/es

LEY 27/2005, de 30 de noviembre, de fomento de la educación y la cultura de paz (BOE n' 287, 01-12-2005).

MARTÍNEZ, M.J. (2003). Educación comparada. Nuevos retos, renovados desafíos. Madrid: La Muralla.

MAYOR, F. \& DE LA CORTE, L. (2002). De la cultura de la violencia a la cultura de paz. Entrevista a Federico Mayor Zaragoza. Cultura y Educación, 14 (4), 359-371.

ONU: Resolución 53/25 de Naciones Unidas adoptada en noviembre de 1998 proclamando el período comprendido entre 2001 y 2010 "Decenio Internacional para una cultura de paz y no violencia para los niños del mundo".

SUARES, M. (1996). Mediación. Conducción de disputas, comunicación y técnicas. Buenos Aires: Paidós.

TORREGO, J.C.(Coord.) (2003). Mediación de conflictos en instituciones educativas. Manual para la formación de mediadores. Madrid: Narcea.

TORREGO, J.C. \& GALÁN, A. (2008). Investigación evaluativa sobre el programa de mediación de conflictos en centros escolares. Revista de Educación, 347, 369-394.

URANGA, M. (1997). Experiencia de mediación escolar en Gernika. Aula de Innovación Educativa, 65, 65-68. 
VAN SLYCK \& STERN, M. (1996). La resolución del conflicto en marcos educativos: evaluación de impacto de los programas de mediación paritarios. En Grover, K; Grosch, J.W. y Olczad, P.V. La mediación y sus contextos de aplicación. Una introducción para profesionales e investigadores (pp. 305-323). Barcelona: Paidós Ibérica.

VIANA-ORTA, M-I (2011). La mediación en el ámbito educativo en España. Estudio comparado entre Comunidades Autónomas. Valencia: Servicio de Publicaciones de la Universitat de València.

XUNTA DE GALICIA. Plan Integral de Mejora de la Convivencia Escolar en Galicia. Consultado el 24-01-2011 en: https://www.edu.xunta.es/convivencia/plan_integral_convivencia_pdf/plan_integr al\%20.pdf

\section{Correspondencia con la autora}

María-Isabel VIANA-ORTA

Departamento de Educación Comparada e Historia de la Educación

Universitat de València, España

Avda. Blasco Ibáñez, $n^{\circ}$ 30. 46020-Valencia

M.Isabel.Viana@uv.es 